\title{
Miomectomía en gestante de 14 semanas: a propósito de un caso
}

\author{
Ima Moslemi Y. ${ }^{1}$, Verónica Alarcón C. ${ }^{1}$, Zandra Soto P. ${ }^{1}$, Karina Mancisidor S. ${ }^{1}$, Santos \\ Sanchez A. ${ }^{2}$, Luis Peñalosa $R^{2}$ \\ ${ }^{1}$ Residente de Ginecología y Obstetricia, Hospital Clínico Universitario de Salamanca. ${ }^{2}$ Servicio de Ginecología y \\ Obstetricia, Hospital Clínico Universitario de Salamanca. Salamanca, España.
}

\section{RESUMEN}

Antecedentes: La prevalencia de los miomas uterinos en la gestación es de 0,3 a 2,6\% de los cuales un $10 \%$ se complican durante el embarazo. El manejo quirúrgico de los miomas en la gestación se reserva solo para los casos complicados. Caso clínico: Paciente de 36 años, primigesta, que consulta a las 11 semanas de gestación por cuadro de distensión abdominal, edema en extremidades inferiores y sangrado vaginal escaso. El examen físico y la ecografía evidencia un mioma de $23 \mathrm{~cm}$ de diámetro y saco gestacional con embrión vivo a nivel de hipocondrio izquierdo. En controles posteriores la paciente empeora clínicamente debido al crecimiento del mioma, presentado dolor abdominal intenso, aumento de edemas en ambas extremidades inferiores. Los estudios de imagen informan ectasia pielocalicial bilateral y compresión de venas ilíacas. Ante los hallazgos y clínica se realiza miomectomía sin incidencias a las 14 semanas de gestación. El postoperatorio y controles posteriores son normales y se programa cesárea a las 37 semanas de gestación. Discusión: La miomectomía en la gestación conlleva riesgos de hemorragia y aborto. Se reserva para casos puntuales que no respondan al manejo expectante. La recomendación actual y la experiencia indican que se debe realizar en el segundo trimestre de gestación. Conclusión: La miomectomía en la gestación es una técnica que se debe plantear en casos seleccionados y que presenta pocas complicaciones.

PALABRAS CLAVE: Mioma uterino, miomectomía, embarazo

\section{SUMMARY}

Background: The prevalence of uterine fibroids in pregnancy is 0.3 to $2.6 \%, 10 \%$ of which complicate during pregnancy. The surgical management of fibroids in pregnant women is reserved for complicated cases. Case report: The patient is 36 years old, first pregnancy, consulting at 11 weeks of gestation with bloating, edema in the lower extremities and mild vaginal bleeding. Physical examination and ultrasound evidence a $23 \mathrm{~cm}$ diameter fibroid and gestational sac with live embryo in the left upper abdominal quadrant. In subsequent tests the patient worsens clinically due to fibroid growth, presenting intense abdominal pain, increased edema in both lower extremities. Imaging studies report pyelocalyceal bilateral ectasia and compression of iliac veins. Given these findings and symptoms a myomectomy is performed without incidents at 14 weeks of gestation. Postoperative and subsequent tests are normal and caesarean section is preformed at 37 weeks of gestation. Discussion: myomectomy in pregnancy carries risks of bleeding and abortion. It is reserved for cases that do not respond to expectant management. The current recommendation and experience indicate that it has to be performed in the second trimester. Conclusion: Myomectomy in pregnancy is a technique that should be considered in selected cases and has few complications.

KEY WORDS: Uterine fibroid, myomectomy, pregnancy 


\section{INTRODUCCIÓN}

Los miomas son tumores monoclonales que surgen de la musculatura lisa del útero y que tienen características moleculares particulares lo que hace que la tasa de crecimiento intrínseco de cada uno de ellos sea distinto, independiente del tamaño y la localización (1-3). La etiopatogenia exacta de los miomas aun no está del todo dilucidada, pero se sabe que hay una mayor concentración de receptores de estrógenos y progesterona en estas tumoraciones en comparación con el miometrio adyacente, por lo que la exposición prolongada a hormonas esteroidales sexuales afecta su desarrollo y crecimiento: menarquia temprana, embarazo, perimenopausia y obesidad. Además su reducción en la menopausia claramente denota su dependencia a los estrógenos (1).

Los datos de la literatura sugieren que el crecimiento de los miomas en la gestación no está directamente relacionado con los niveles séricos de estrógenos, ya que los miomas tienen un crecimiento importante en el primer trimestre de embarazo, en particular doblan su tamaño a las 6-7 semanas gestacionales, cuando lo estrógenos y la progesterona aun mantienen niveles bajos. Después, tras una fase de desaceleración en su crecimiento en la segunda mitad del embarazo, disminuyen su tamaño en el tercer trimestre (4).

Al parecer la hipertrofia e hiperplasia de las células miometriales es por una serie de hormonas, citoquinas y factores de crecimiento producidas por la unidad fetoplacentaria (5), a través de mecanismos directos e indirectos. Independientemente hay suficiente evidencia para decir que los estrógenos son presumiblemente los promotores primarios del crecimiento de los miomas y los progestágenos estarían involucrados en su mantenimiento y crecimiento (6).

La prevalencia de los miomas uterinos en la gestación es de 0,3 a 2,6\% de los cuales un 10\% se complican durante el embarazo (7). El manejo quirúrgico de los miomas en el embarazo se reserva solo para los casos complicados ya que siempre se aboga por el manejo conservador que consiste en reposo, hidratación y analgesia.

Las complicaciones más frecuentes son aborto, dolor pélvico, desprendimiento prematuro de placenta normo inserta (DPPNI), hidronefrosis, rotura prematura de membranas (RPM), parto prematuro, restricción de crecimiento intrauterino, distocias de presentación y hemorragias posparto (8). La prevalencia de estas complicaciones aumenta cuando el mioma es múltiple, retroplacentario y mayor de 3,6 $\mathrm{cm}$ de diámetro (7). También aumenta la tasa de cesáreas e histerectomías obstétricas.

En casos seleccionados la miomectomía mejora el resultado obstétrico cuando se compara con el manejo conservador (9). El objetivo de esta comunicación es presentar el caso del manejo de un mioma de gran volumen durante la gestación.

\section{Caso clínico}

Paciente de 36 años, primigesta, con antecedentes personales de hipotiroidismo en tratamiento que consulta en urgencias por cuadro de distensión abdominal, edema en extremidades inferiores y sangrado vaginal escaso a las 11 semanas de amenorrea. A la exploración se evidencia tumoración que llega hasta nivel supraumbilidad, dura y pétrea. Cérvix desviado a la izquierda de muy difícil visualización, sin sangrado activo. Edema leve en extremidades inferiores. En la ecografía abdominal, gran mioma que ocupa toda la pelvis menor de $23 \times 20 \times 13 \mathrm{~cm}$ y saco gestacional con embrión que corresponde a la amenorrea, latido cardiaco positivo, localizado a nivel de hipocondrio izquierdo.

Se inicia controles de la paciente en la consulta de alto riesgo obstétrico evidenciando empeoramiento del cuadro clínico debido al crecimiento del mioma, manifestado por dolor abdominal y dolor asociado al aumento del edema en extremidades inferiores. Se solicita RNM a las 13 semanas de amenorrea que informa: tumoración pélvica compatible con mioma (Figura 1). Ectasia pielocalicial bilateral y compresión de venas iliacas externas. Eco doppler de extremidades inferiores: sin evidencia de trombosis a nivel de venas femorales comunes, flujo lento en iliacas externas. Se decide iniciar profilaxis antitrombótica con bemiparina sódica 3500 UI subcutáneo al día.

Ante los hallazgos de gestación de 14 semanas y mioma uterino con compromiso vascular se plantea intervención quirúrgica previo consentimiento de la paciente. Se procede a la miomectomía de mioma intramural subseroso en borde derecho uterino, de $27 \mathrm{~cm}$ de diámetro (Figura 2). Se inyectan 20 cc de adrenalina diluida en suero (1 mg adrenalina en $250 \mathrm{cc}$ de suero fisiológico) en la capa subserosa donde se realiza incisión inicial y se procede a la decapsulación del mioma sin incidencias, sin entrar a la cavidad endometrial (Figura 3). Sutura discontinua por capas y se deja un hemostático en el lecho quirúrgico (matriz formada por gránulos de gelatina de origen bovino y de trombina humana en presentación de gel) (Figura 4). La anatomía patológica posteriormente informa leiomioma.

El postoperatorio fue de curso normal. Se realizó ecografía previa al alta evidenciando embrión único, vivo y biometría que correspondía a la amenorrea. Placenta en cara posterior, líquido amniótico normal. La paciente continuó controles prenatales con un embarazo de curso normal. Se programó cesárea en la semana 37 de gestación extrayendo recién nacido masculino de $3020 \mathrm{~g}$ en presentación transversa, sin incidencias. 

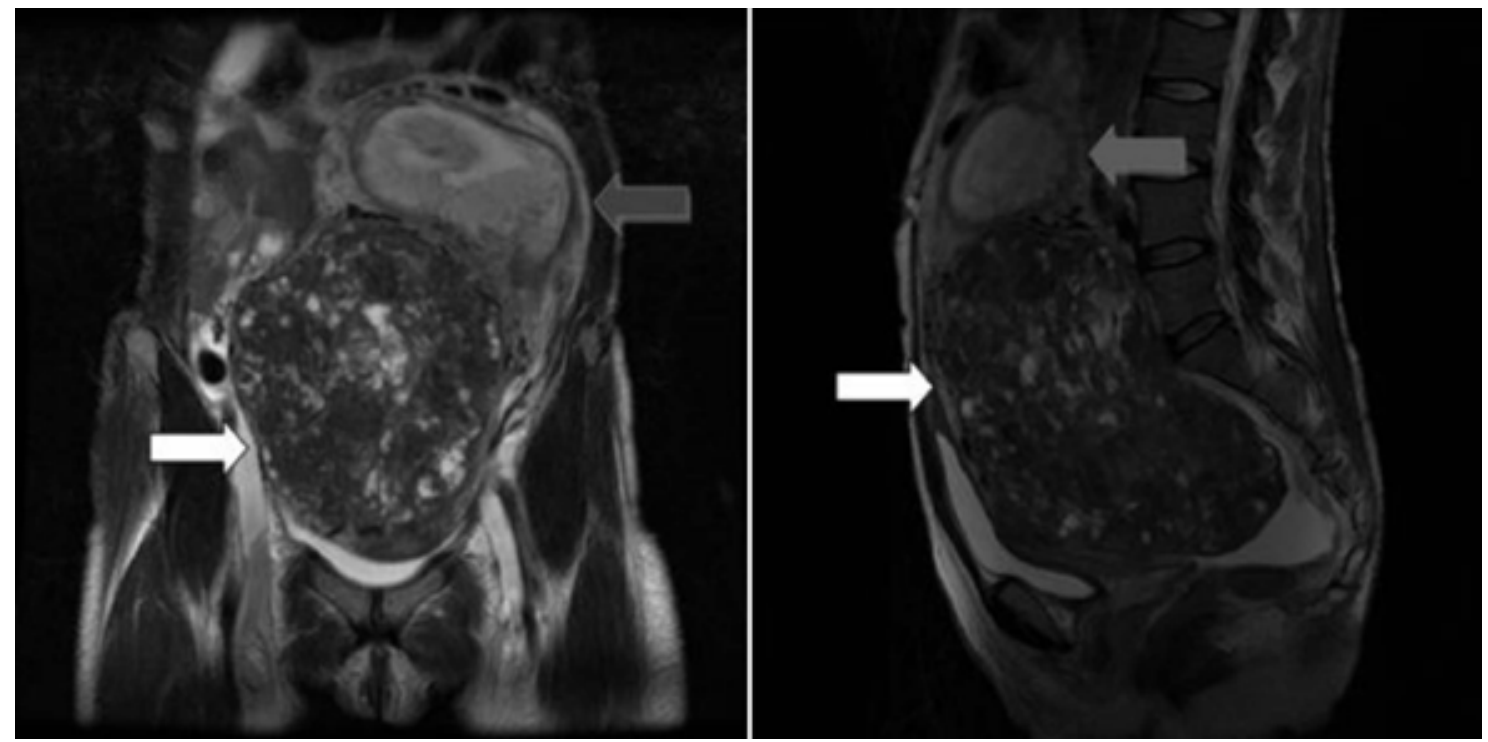

Figura 1. La flecha gris señala saco gestacional con embrión de 13 semanas, la flecha blanca señala mioma uterino previo de gran tamaño (RNM).

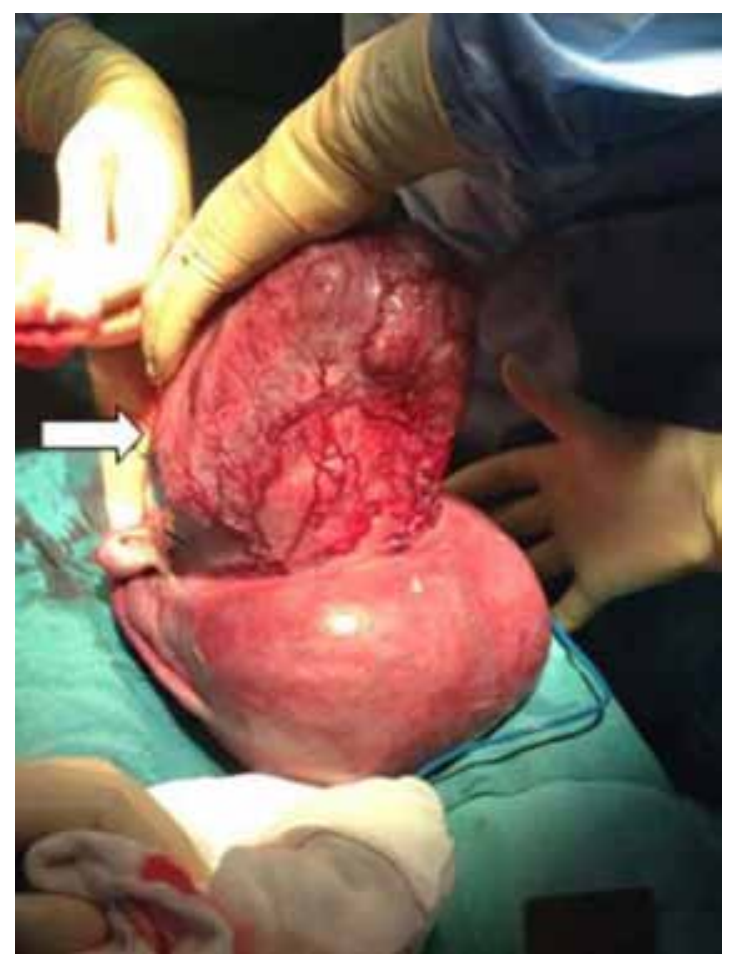

Figura 2. Se procede a miomectomía previa inyección subserosa de adrenalina y tras extracción de útero grávido de la cavidad abdominal.

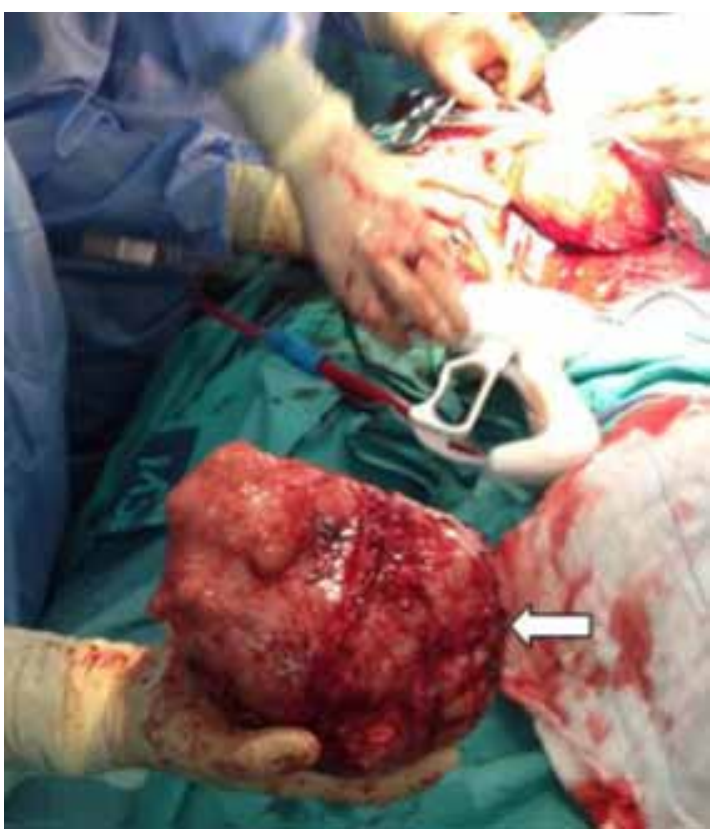

Figura 3. Se realiza decapsulación sin incidencias, extrayendo pieza quirúrgica. 


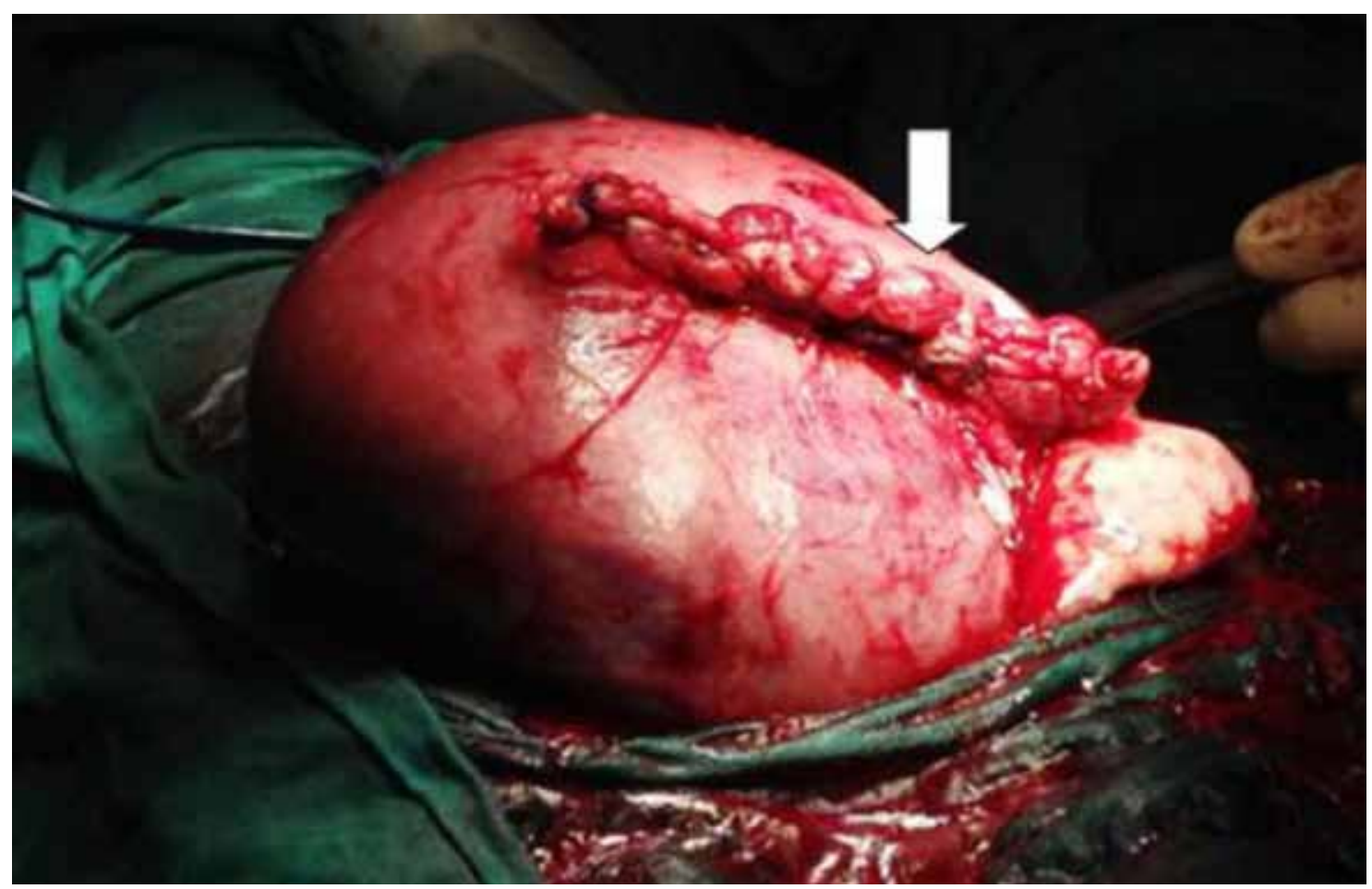

Figura 4. Sutura por capas con puntos discontinuos y aplicación de hemostático en gel en lecho quirúrgico.

\section{DISCUSIÓN}

Los miomas son los tumores sólidos que con más frecuencia se detectan durante la gestación (9) y pueden ser causa de resultado obstétrico desfavorable, aunque los miomas de gran tamaño, previos y de crecimiento rápido son más raros. La revisión de la literatura indica que las complicaciones oscilan entre un 10 a un $40 \%$, pero esto no es real ya que la incidencia de los miomas en la gestación es infraestimada y las complicaciones sobrestimadas (10).

El dolor es una de las complicaciones más frecuentes que en ocasiones requiere hospitalización para su manejo y es más frecuente en miomas de más de $5 \mathrm{~cm}$. El dolor se produciría por una disminución de la perfusión en el contexto de un rápido crecimiento que llevaría a isquemia y necrosis. Es debido a esto que generalmente se desencadena a finales del primer trimestre o inicios del segundo trimestre que es el periodo de mayor crecimiento (10) y que coincide con el caso de nuestra paciente.

La miomectomía conlleva riesgos de hemorragia y aborto por lo que solamente se reserva para casos puntuales que no respondan al manejo expectante (11). La recomendación actual y la experiencia indican que se debe realizar en el segundo trimestre de gestación aunque hay casos reportados en el primer trimestre. Incluso estas pacientes tendrían mejor resultado obstétrico que las tratadas de forma expectante (12).

Mollica y cols (13) realizaron un estudio con 106 gestantes con miomas uterinos comparando tratamiento quirúrgico vs expectante. La miomectomía se realizó en 18 mujeres con rápido crecimiento del mioma y dolor, miomas de más de $5 \mathrm{~cm}$ en segmento uterino o retroplacentarios. Se evidenció mayores tasas de aborto, parto prematuro e histerectomía obstétrica en el grupo de manejo expectante.

Las recomendaciones actuales respecto a miomectomía y gestación nos indican que el tiempo ideal para lograr la cicatrización del miometrio son 12 meses y así disminuir el riesgo de rotura uterina que es de un 0,2\% en estas pacientes.

En nuestro caso se programa cesárea a la semana 37 por presentación transversa y por disminuir el riesgo de rotura uterina que aumenta en las últimas semanas de gestación y evitar que se desencadene trabajo de parto espontaneo. Aun no existen recomendaciones ni protocolos sobre como finalizar la gestación en las pacientes que se realizan miomectomías en segundo trimestre del embarazo. 


\section{CONCLUSIÓN}

La revisión de la literatura indica que la miomectomía en la gestación es una técnica que se debe plantear en casos seleccionados y que presenta pocas complicaciones. La intervención se debe programar con un equipo con experiencia y la vía de abordaje y la técnica se debe consensuar según las características del mioma y los antecedentes de la paciente. Aun se necesitan más estudios para determinar los criterios de inclusión y la seguridad del procedimiento para llegar a un consenso.

\section{REFERENCIAS}

1. Ciavattini A, Di Giuseppe J, Stortoni $P$, et al. Uterine fibroids: pathogenesis and interactions with endometrium and endomyometrial junction. Obstet Gynecol Int 2013; 2013:173184.

2. Lobel MK, Somasundaram P, Morton CC. The genetic heterogeneity of uterine leiomyomata. Obstet Gynecol Clin North Am 2006;33:13-39.

3. Wei JJ, Chiriboga L, Mittal K. Expression profile of the tumorigenic factors associated with tumor size and sex steroid hormone status in uterine leiomyomata. Fertil Steril 2005;84:474-84.

4. Benaglia L, Cardellicchio L, Filippi F, et al. The rapid growth of fibroids during early pregnancy. PloS One 2014;9:e85933.

5. Parker WH. Etiology, symptomatology, and diagnosis of uterine myomas. Fertil Steril 2007;87:725-36.
6. Segars JH, Parrott EC, Nagel JD, et al. Proceedings from the Third National Institutes of Health International Congress on Advances in Uterine Leiomyoma Research: comprehensive review, conference summary and future recommendations. Hum Reprod Update 2014;20:309-33.

7. Suwandinata FS, Gruessner SEM, Omwandho COA, Tinneberg HR. Pregnancy-preserving myomectomy: preliminary report on a new surgical technique. Eur $\mathrm{J}$ Contracept Reprod Health Care 200813:323-6.

8. Leach K, Khatain L, Tocce K. First trimester myomectomy as an alternative to termination of pregnancy in a woman with a symptomatic uterine leiomyoma: a case report. J Med Case Rep 2011;5:571.

9. De Carolis S, Fatigante G, Ferrazzani S, Trivellini C, De Santis L, Mancuso S, Caruso A. Uterine myomectomy in pregnant women. Fetal Diagn Ther 2001;16:116-9.

10. Ouyang D, Economy K, Norwitz E. Obstetric Complications of fibroids. Obstet Gynecol Clin North Am 2006;33(1):153-69.

11. Domenici L, Di Donato V, Gasparri ML, Lecce F, Caccetta J, Panici PB. Laparotomic myomectomy in the 16th week of pregnancy: a case report. Case Rep Obstet Gynecol 2014;2014:154347.

12. Celik C, Acar A, Ciçek N, Gezginc K, Akyürek C. Can myomectomy be performed during pregnancy? Gynecol Obstet Invest 2002;53:79-83.

13. Mollica G, Pittini L, Minganti E, Perri G, Pansini F. Elective uterine myomectomy in pregnant women. Clin Exp Obstet Gynecol 1996;23:168-72. 